\title{
Static Integration Based on Stochastic P-Time Petri Nets of Maintenance Scheduling for Railway Transport Equipment
}

\author{
Anis M'halla $\mathbb{D}$ \\ Laboratory of Automation, Electrical Systems Environment (LAESE), National Engineering School of Monastir (ENIM), \\ Monastir, Tunisia \\ Correspondence should be addressed to Anis M’halla; anis_mhalla2003@yahoo.fr
}

Received 6 May 2021; Revised 6 July 2021; Accepted 30 July 2021; Published 9 August 2021

Academic Editor: Giulio E. Cantarella

Copyright $(2021$ Anis M'halla. This is an open access article distributed under the Creative Commons Attribution License, which permits unrestricted use, distribution, and reproduction in any medium, provided the original work is properly cited.

In transport systems, all equipment requires maintenance, which directly affects the machine's availability and consequently the planned transport schedule. The purpose of this paper is to carry out a method for integrating recovery jobs in railway systems. The proposed method allows the insertion of preventive and corrective maintenance operations when the transport equipment is available in order to minimize periods of inactivity, avoid catastrophic scenarios, and maintain stability and safety of the studied networks. A computing algorithm, allowing insertion of the planned recovery tasks in periods of metro availability, without changing the initial scheduling solution, is established. Finally, we illustrate the implementation of the proposed approach on Tunisian Sahel railway transport networks.

\section{Introduction}

National railways are typically large and sophisticated systems. Their network infrastructure usually contains extended sections, stations, and other support activities. Railway infrastructure assets have a very long service life, although in principle deteriorate, and thus interventions are necessary, which will cause delays, damage, and dangers.

As the railway industry evolved, maintaining equipment running without failure became crucial. Traffic disturbances due to transport equipment breakdown were no longer acceptable due to the growing demand. In railway transport systems, the initial concept of equipment maintenance policy was reactive maintenance. Recently, corrective maintenance was replaced by scheduled maintenance resulting in better equipment availability. There is a relatively small amount of literature on transport network scheduling problems which considers the issues associated with a lack of equipment. However, in most practical transport environments, there is always some uncertainty surrounding the availability of trains, such as obstacles on the tracks, malice, social movements, the absence of staff, downtime of equipment, interrupted customer service, and so on.
Scheduling involves the allocation of resources to tasks over time subject to temporal constraints. In some cases, scheduling reveals periods of inactivity of transport equipment in railway networks systems. The objective of this paper is to propose, taking advantage of these periods of inactivity, an approach allowing the insertion of preventive and corrective maintenance tasks.

The problem of maintenance scheduling with the presence of disruptions is of wide importance for the successful implementation of real scheduling systems. However, in recent years, only a few studies have been published in this area. In this paper, we are interested in maintenance scheduling, based on stochastic P-time Petri nets in railway network, when a sudden event occurs. We propose a method to insert additional recovery jobs in railway transport network systems. The goal of the proposed method is the introduction of preventive (inspection, visit, control, and so on) or urgent tasks, taking into account all transport constraints.

Based on this concept, there are two methods of introducing additional tasks in scheduling. The first method is known as "static insertion" and consists of the introduction of a set of tasks in the availability of the machines; the second 
one entitled "dynamic insertion" is designed to recreate the schedule if an event changes the data. The ultimate goal is to optimize the impact of this integration on traffic management to avoid degradation of the transport service and minimize railway transport cost.

This paper is organized as follows. Section 2 uses the scientific literature to explain the position of the proposed scheduling approach in the state of the art. A description of railway transport system is detailed in Section 3. Section 4 constitutes the contribution of this paper. A computing algorithm allowing the insertion of recovery jobs in periods of transport equipment availability without changing the initial scheduling solution is established. In Section 5, the proposed maintenance scheduling approach is demonstrated using the case study of Tunisian railway network. Finally, the conclusion and extensions to this method are outlined in Section 6.

\section{State of the Art}

In railway transport networks, failure cannot be avoided, especially in terms of modern electric multiple unit (EMU). Keeping these units in perfect condition is of importance to researchers and manufacturers. There have been few studies which consider integrated maintenance scheduling (IMS) in order to increase availability of the EMU. In this context, a few research projects were instigated regarding the maintenance of railway transportation systems in order to save time and improve rail service quality [1-5].

2.1. Maintenance Policy. In order to predict rail defects, the authors in [6] presented a new strategy that incorporates prediction with inspection and maintenance scheduling activities. A Markov decision process model which allows the prediction of maintenance scheduling is introduced.

Yaghini et al. [7] considered the problem of the shortterm scheduling of track maintenance activities after the train schedule and maintenance activities have been planned. The main goal is to minimize maintenance costs. The proposed maintenance scheduling model is based on a twostage heuristic technique. The first stage consists of the generation of a realistic solution, and the second stage is the implementation of simulated annealing and a local search.

A risk-based decision support system to schedule the predictive maintenance activities was proposed by Consilvio et al. [4]. In such a framework, the interventions are planned by taking into account the forecast degradation state of railway assets and performed when a given threshold is reached, thus minimizing the probability of both sudden and unnecessary operations.

2.2. Search Algorithms. Other authors [8] considered autonomous scheduling of maintenance jobs. They presented a design strategy using a novel integrated system for automatic job scheduling. A proof of concept demonstrator was developed to validate the system principle and to test algorithm functionality.
The results are promising, since the application of the new approach offers further scope for research and commercial exploitation.

Xie et al. [9] developed a customized stepwise algorithm to solve a vehicle routing problem with time windows (VRPTWs) for rail maintenance routing and scheduling problems (RMRSPs). A number of numerical experiments are performed to demonstrate that the proposed algorithm works very effectively and significantly.

In order to attenuate travel costs and penalties associated with waiting times and maintenance activities, Andrés et al. [10] proposed a model using branch and column generation approaches for the railway routing problem, which determines specific paths for every train, given possible maintenance planning.

In order maximize the life cycle quality level of the trackbed considering different levels of reliability, Bressi et al. [1] proposed a methodology to minimize the life cycle maintenance costs. Probabilistic degradation models are developed for predicting the evolution of the railway track condition over time. Afterwards, a genetic algorithm-based optimization procedure is applied for obtaining a set of optimal solutions taking into account several constraints.

2.3. Simulation Methods. In [11], the authors support the track recovery planning problem with three separate settings as three variants of the capacitated arc routing problem with fixed cost (CARPF), which is solved by converting them into three-node routing problems. The proposed approach is demonstrated by employing a case study of a neighborhood of the Dutch regional network.

Dao et al. [12] proposed an optimization model that is aimed at finding the best maintenance schedule for multiple components in a railway track to minimize the total cost in the planning horizon.

In this approach, all relevant costs are formulated including recovery cost, fixed track possession cost, and cost associated with the consequences of maintenance time on the train operation.

The authors in [3] presented an integer linear programming mathematical model, which schedules maintenance tasks for a given period, within the normal rolling-stock service operations. This model was applied to a case study, and a reliability analysis was conducted, assessing the impact of uncertainty associated with maintenance durations in the optimal value for the objective function, as well as in the feasibility of such a maintenance schedule.

All previous works are different from our work. The objective of this paper is trying to take advantage of the rolling stock inactivity periods to launch preventive and corrective maintenance actions taking into account railway traffic constraints. This is done by the study of the rolling stock age and the travelling cycle time. For the stage of adding maintenance tasks, we propose an inserting algorithm. The proposed algorithm allows to integrate unforeseen maintenance actions and to make real-time scheduling of these recovery acts with respect to transport scheduling constraint. The main purpose is minimizing the 
interruptions in the equipment operating/service schedule and to maximize the utilization of the maintenance resources. To the best of our knowledge, such scheduling method has been never formalized for railway transport networks.

This paper contributes to the state of the art of railway maintenance scheduling problem and addresses a situation where the possession time is restricted. By investigating the presented maintenance strategy, this paper can provide an answer for infrastructure managers of complex railway networks where there is a growing demand for using the track for train-path operation and there is rising pressure to extend operation time, thus reducing the infrastructure accessible for maintenance.

The contributions of the present paper are as follows:

(i) Development of a new recovery approach based on the study of rolling stock age and the travelling cycle time.

(ii) For the stage of maintenance scheduling, we propose an inserting algorithm allowing the introduction of additional maintenance tasks in the availability of the machines without changing the initial scheduling solution.

\section{Proposed Method}

Given a set of maintenance tasks, it is interesting to seek out good schedules for all jobs to reduce disruptions and at the same time accommodate unexpected events. Thus, the impartial objectives of maintenance scheduling are to attenuate the interruptions in the equipment operating/service schedule and to avoid disastrous scenarios by retaining stability and safety of studied railway networks.

A scheduling method consists of the development of a procedure which allows the insertion of a provisional job into the solutions found previously, while optimizing a certain number of criteria. We distinguish two insertion methods: static and dynamic methods.

Static insertion methods allow the following:

The insertion of the operations of the forecast requests into the availability of machines, in order to minimize the periods of inactivity and to increase the productivity rate.

The postponement of certain operations, if the uptime of the machines is insufficient, in order to minimize the total production time of all orders.

The dynamic methods allow the following:

Reordering as and when orders arrive or when job characteristics change. This is the case, for example, when it is necessary to create machine availabilities by shifting nonurgent operations to insert urgent orders or requests that have tight delivery dates.

The main contribution of the paper is the static insertion of a set of maintenance jobs in the availability of the transport equipment in a railway networks system. In this context, a computing algorithm allowing the insertion of a set of maintenance actions is proposed.

3.1. Age-Dependent Maintenance Planning Based on SP-TPN Model. Many transport systems deteriorate with age and are also subject to random failures. Therefore, for more expensive and critical systems, it is important to determine the optimal maintenance and replacement policy to reduce the catastrophic breakdown risks and operating costs. The main objective of this section is to integrate cycle time of rolling stock and the EMU age to decide about the selective maintenance decision of the railway transport equipment.

In our approach, the decision variables are the cycle time of travelling circuits and the machine age. In transport systems, each equipment machine is subject to a maximum age $M$. Machine age is defined as the cumulated operating time (travelling and staying times). If the machine age reaches $M$, various maintenance policies have to be carried out right after the completion of the operating operation.

In this section, we try to prove that the cyclic scheduling technique can be well applied to the maintenance of a medium-sized railway transport network. Cyclic scheduling is defined as a set of activities that can be repeated for infinite number of times. More precisely, if $X(n)$ is the starting time (or ending time) of one transport activity and $n$ means the repeat numbers, then there is a constant $C$ (called the cycle time which is the inverse of the periodic output rate) and one integer $K$ such that

$$
X(n+k)=X(n)+k * C, \quad \text { for } n \in \stackrel{\vee}{c}, k \in \stackrel{\vee}{c}, C \geq 0
$$

It is assumed that, in our work, only the 1-cyclic $(k=1)$ scheduling technique is studied. Factually, the 1-cyclic operation transport can be described as a SP-time Petri net. Here, the SP-TPN is a P-time Petri net in which each place is assigned with a time window.

\subsubsection{Problem Description}

The main objective of this paper is to integrate failure rate, travelling cycle time, and the EMU age to decide about the selective maintenance decision of the rolling stock in railway transport networks.

In railway networks, the transport equipment age is equal to the cumulated travelling time. After doing a number of transport operations, each EMU requires a time for maintenance. The conceptual idea of this paper is represented in Figure 1.

\subsubsection{Framework of the Proposed Maintenance Approach}

Replacement over Age. In railway transport networks, each rolling stock has $N_{j}$ number of weekly travel operations, and each operation can be performed on more than one suitable EMU with different processing time.

The EMU age is given by the following expression: 


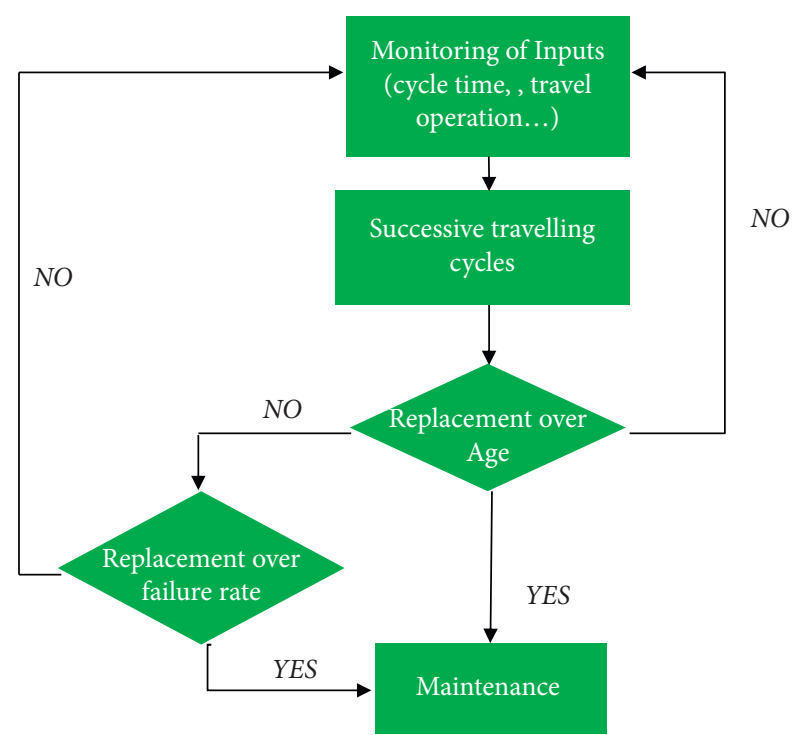

Figure 1: The flow sheet for the maintenance of seaport container terminal.

$$
A_{M F}=\sum_{j=1}^{m} \sum_{i=1}^{N} N_{i} *\left(T E_{i j}-T S_{i j}\right)+M T_{M F}
$$

Objective. The objective of this function is computing the EMU cumulated operating time. The EMU age expression has as input number of travel operation $\left(N_{i}\right)$, travel duration, and maintenance time (MT). In Sahel railway networks, each EMU can perform various travel operations with different operating lead times. After doing a number of operations, each transport equipment requires a time for maintenance.

Variables. The EMU age equals the cumulated travelling time and cumulated time allocated to the maintenance. Table 1 shows the variables that define the expression of the EMU age.

The EMU age is defined by the time constraints and the convex hull of the extreme points $A_{M f}$ generated and active at iterations " $\mathbf{i}^{\prime \prime}$ and " $\mathbf{j}$." The age function is a convex combination of the extreme parameters like travel duration and maintenance time .

Referring to the above definition, each EMU is subject to a maximum machine age $A$. The machine age is defined as the cumulated travel time. If the EMU age reaches $A$, maintenance has to be carried out right after the completion of the operating operations. After maintenance, the machine age will be reset to 0 as shown in Figure 2.

3.2. Determination of Sojourn Time and Cycle Time. In TNRC, each train should endure maintenance checks after a particular number of hours or distance (kilometers of travelling time). According to the results reported by the SCADA of the TNRC, each EMU guarantees 25 daily scheduled traffic between Monastir and Sousse with a mean of 30-minute frequency.
According to times established by the regulations, the total weekly cycle time of each EMU is 5250 minutes with the demanded traffic rate. But sometimes, the metros are in waiting buffer without running. So, the age of the rolling stock is relative to the sojourn time in path places that represent travelling and staying times.

Taking into account the weekly cycle time, maintenance checks happen at the corresponding TNRC depots that have maintenance facilities among the network.

In this section, we have proposed an approach for maintenance based on the study of the rolling stock age and the travelling cycle time. The next step is the implementation of these recovery jobs in a railway transport networks. The scheduling method must allow inserting preventive and corrective maintenance operations when the EMU is available in order to increase the railway traffic rate. In this context, a computing algorithm allowing insertion of the projected jobs in transport equipment availability periods, without changing the initial scheduling solution, will be presented in the next section. An implementation of the scheduling method to Tunisian railway network will be established.

\subsection{Static Insertion of Maintenance Actions}

3.3.1. Principle. The static insertion proposes a new sequence of operations " $T_{i j}$ " (operation $i$ associated to a job $j$ ) for a problem defined by the planning layer (Figure 3). The goal of static insertion is to maximize the availability of transport equipment and to decrease cost of unexpected failures. Inserting of a task " $T_{i j}{ }^{\prime \prime}$ in the initial scheduling is in accordance with the following conditions (Figure 3):

No change in the allocation of scheduled operations.

No violation of the end dates of the programmed operations.

In this paper, an application of the static integration approach to the management of maintenance operations in railway transport system is proposed.

3.3.2. Notations. The following notations are used to describe the problem studied throughout the paper:

$h$ : index for metro, $h=1, \ldots, H$, where $H$ is the number of metro (EMU)

$j$ : index for travelling time, $j=1, \ldots, J$, where $J$ is the number of travelling time

$i$ : index for maintenance operation (MO), $i=1, \ldots, I$, where $I$ is the number of operation

$S T_{j}$ : starting travelling time $j$ of a metro $h$

$E T_{j}$ : ending travelling time $j$ of a metro $h$

$\mathrm{ST}_{\mathrm{RO}}$ : starting time of recovery operation

$\mathrm{ET}_{\mathrm{RO}}$ : ending time of recovery operation

Ah: availability interval of machine $h$

$P_{\mathrm{RO} h}= \begin{cases}1 & \text { possibility of insertion of RO for EMU } h \\ 0 & \text { otherwise }\end{cases}$ 
TABLE 1: Formulation of problem.

\begin{tabular}{lc}
\hline$M f$ & Number of EMU $(M)$ in railway transport network $f$ \\
$N i$ & Number of travel operation $i$ of total weekly travel operation $j$ \\
$T S_{i j}$ & Starting time of travel operation $i$ of weekly travel operation $j$ \\
$M T_{M f}$ & Maintenance time allocated to a transport equipment (EMU) $M$ in transport network $f$ \\
$A_{M f}$ & Age of EMU in transport network $f$ \\
$j$ & Index of travel, $j=1, \ldots, m$, where $m$ is the number of weekly travel operations \\
$T E_{i j}$ & Ending time of travel operation $i$ of weekly travel operation $j$ \\
$A$ & Maximum EMU age \\
\hline
\end{tabular}

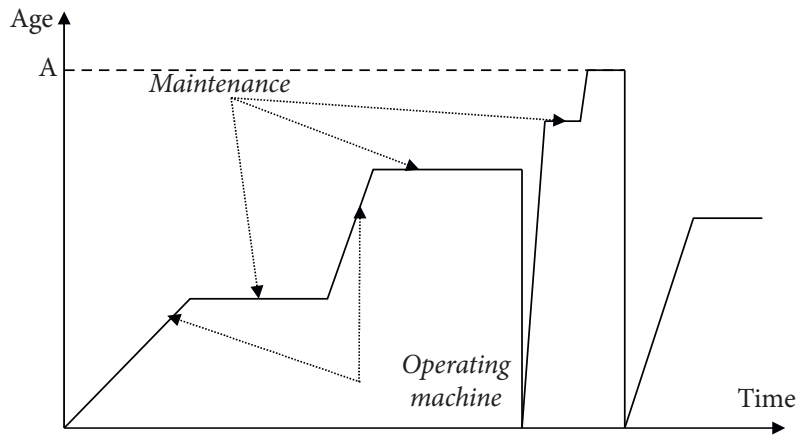

FIgUre 2: Modelling of the maximum EMU age $A$.

$$
O= \begin{cases}\sup _{h}\left(\sum_{j=1}^{J}\left(E T_{j}-S T_{j}\right)\right) & \text { if job j occupies largest time slot on EMU } h \\ 0 & \text { otherwise }\end{cases}
$$

\subsection{Algorithm (Procedure of Static Insertion)}

3.4.1. Purpose. To insert the desired maintenance task in machine availability, without changing the existing scheduling solution, a recursive algorithm based on the priority to the EMU with highest operating lead time (HOLT) is proposed (Algorithm 1).

\section{Case Study: Description and Discussion}

\subsection{Tunisian Railway Network}

4.1.1. Presentation. Tunisian National Railways Company (TNRC) is a state owned company, which is responsible for operation, maintenance, and management of national rail network. The TNRC operates 11 lines which link major cities of Tunisia including the Sahel rail network. This line mainly links the cities and agglomerations of Sahel from Monastir to Sousse. It begins at the Sousse Bab Jedid station, crossing through Sahline to Monastir station by serving the University campus (Figure 4). With a mean frequency of 30 minutes, Sahel Metro guarantees a daily scheduled traffic between 5:00 and 10:00 PM.

4.1.2. Modelling of the Railway Transport System. In the transport system under consideration, the travelling time and staying time are interval valued. So, any deviations from the allowed lower and upper bounds will cause defective traffic disturbances. Stochastic $P$-time Petri nets (SP-TPNs) are practical tools for modelling the studied railway system whose travelling times are not precisely given.
4.1.3. Single Directional Segment Model. Figure 5 shows a part of the $P$-time Petri net modelling a single directional segment (red places (resp. white places) representing the stations (resp. the paths between two stations)).

To ensure safety of the rail network, some monitoring places (yellow places $P_{185}$ and $P_{178}$ ) have been added (Figure 6). To ensure safety of the railway network, some monitoring places (yellow places $P_{185}$ and $P_{178}$ ), have been added (Figure 6). The role of these places is the avoidance of metro collision: In fact, if a metro " $M 1$ " parks at a station $P_{57}$, $M 1$ cannot be caught up by a metro " $M 2$ " since the firing of the transition $T_{57}$ depends to the presence of two tokens, respectively, at places $P_{178}$ and $P_{57}$."

Figure 7 shows a SP-time Petri net $(S)$ modelling the studied transport system. The acquired $S$ is used to study the online maintenance scheduling of the Tunisian Sahel railway system. On this graph, the time associated to the places represent the durations of travel time between two successive stations. The evolution of the token represents the circulation of the trains on the railway. The track from Monastir to Sousse is represented by the places $P_{45}$ to $P_{63}$ (resp. $P_{63}$ to $P_{45}$ ).

To identify the parameters of the SP-TPN model, a set of real data is taken into account (see Table 2), which refers to the circulation of train between Monastir and Sousse Stations, during July 2020. The measurements have been reported by the SCADA of the TNRC. The SCADA system allows controlling traffic, monitoring, real-time processing, and recording events. For each place of $S$, we denote $\left[L_{i j}, q_{i j}^{e}, H_{i j}\right]$ the lower bound of the time window, the expected sojourn time of tokens, and the upper bound of the time window, respectively. The full set time intervals of travelling and parking operations (Figure 7) are summarized in Table 2 (u.t: unit time).

\subsection{Cyclic Scheduling of the Studied Railway Network.} The movement of a train in a railway network can be seen as the successive occupation of network points by the metro during an interval of time (Figures 8 and 9). These two figures are built with reference to preliminary experiments data extracted from the railway line which links Monastir and Sousse cities. We have considered as data the results reported by the SCADA of the TNRC. In the studied railway line, only ten metro types with two convoys are used.

In fact, in the modelling that we have chosen to use, the management of the safety distances between the subways is carried out by a minimum separation times between train using the same track. This is illustrated in Figures 8 and 9. These figures are based on the sojourn time " $q_{i}$ " associated to each place of the SP-TPN model of the studied railway 


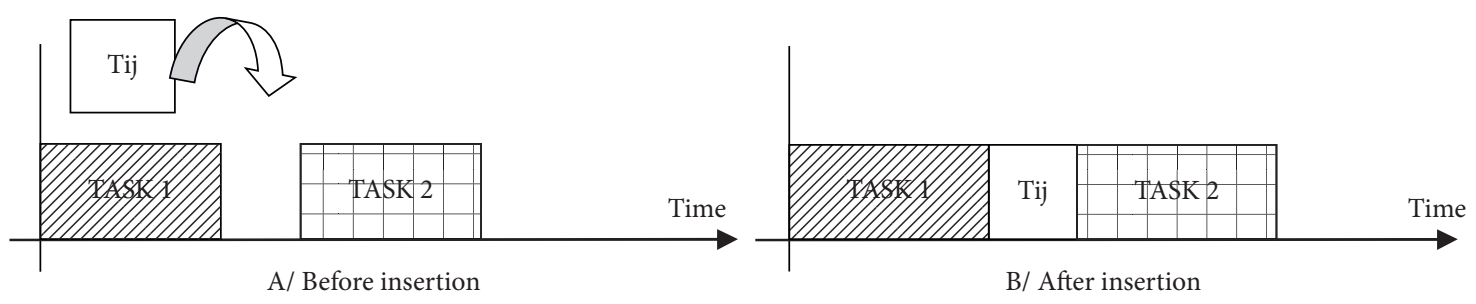

Figure 3: Principle of static insertion. (a) Before insertion. (b) After insertion.

(i) $\operatorname{For}(\mathbf{i}=1 ; \mathbf{i}<\mathbf{I}) ; \mathbf{P}_{\text {ROh }}=0 ; \mathbf{A h}=\varnothing$,

(ii) $\operatorname{For}(\mathbf{h}=1 ; \mathbf{h}<\mathbf{H})$

(iii) If

(iv) \{

(v) $O=\sup _{h}\left(\sum_{j=1}^{J}\left(E T_{j}-S T_{j}\right)\right)$

(vi) $\quad R_{O h} \subset A h$ (the duration of operation insertion corresponding to the period of EMU availability)

(vii) Time constraints are verified

(viii) then

(ix) $\quad P_{R O h}=1$, MLTh $=\sum_{i=1}^{I}\left(E T_{R O}-S T_{R O}\right)($ maintenance lead time for a EMU $h$ )

(x) End

(xi) Insert a maintenance operation on the EMU $h\left(R_{O h}\right)$

(xii) End

Redo the same procedure for all Metros.

Algorithm 1: Procedure of static insertion.

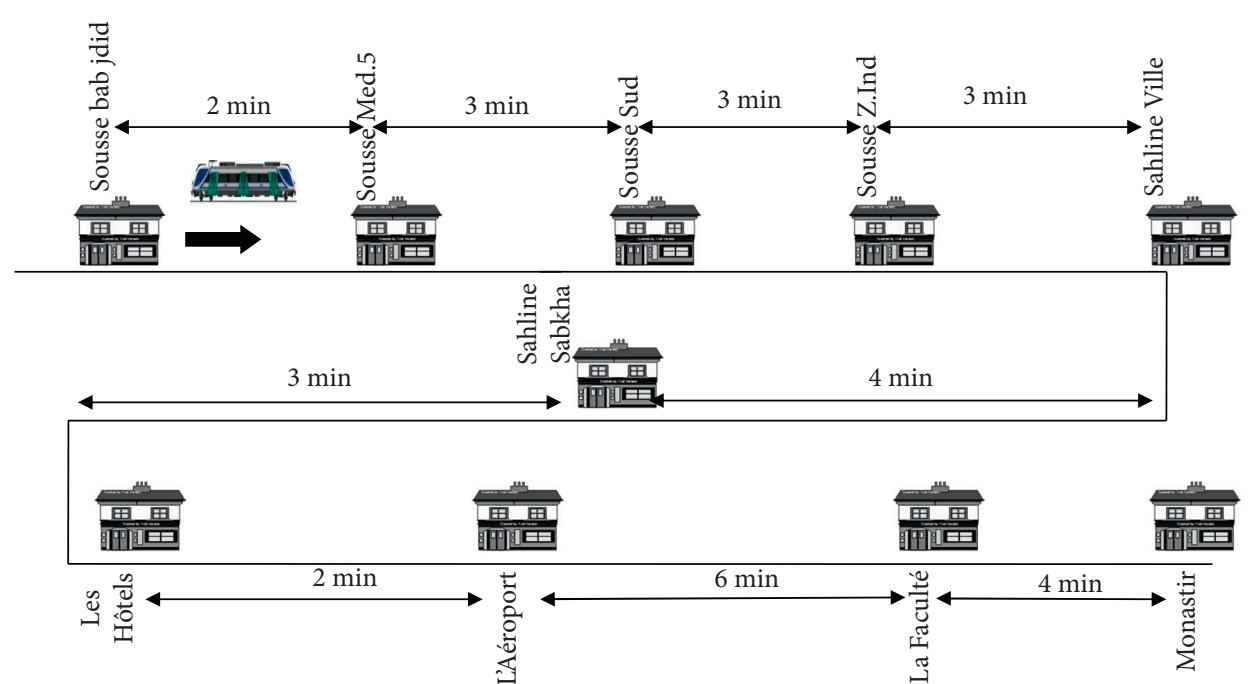

Figure 4: The Sahel railway line.

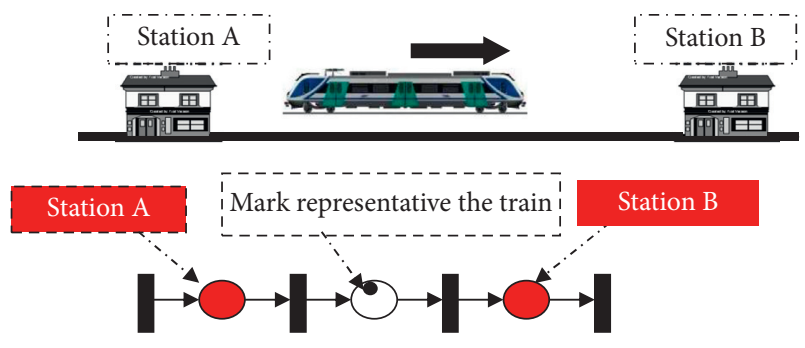

FIgURE 5: Single directional segment model. transport network (see Table 2). Figure 8, for example, shows the travelling and staying time within the studied railway network and can easily model the time windows associated to each travel and sojourn time. For 1-cyclic scheduling, the activities are repetitive during a period cycle time. Thus, it has potential to review the processing activities in one cycle rather than considering the activities within the whole railway transport cycle.

In this case, the itinerary of a train is defined as the order of the points covered during traffic (Figure 7). The traffic entry 


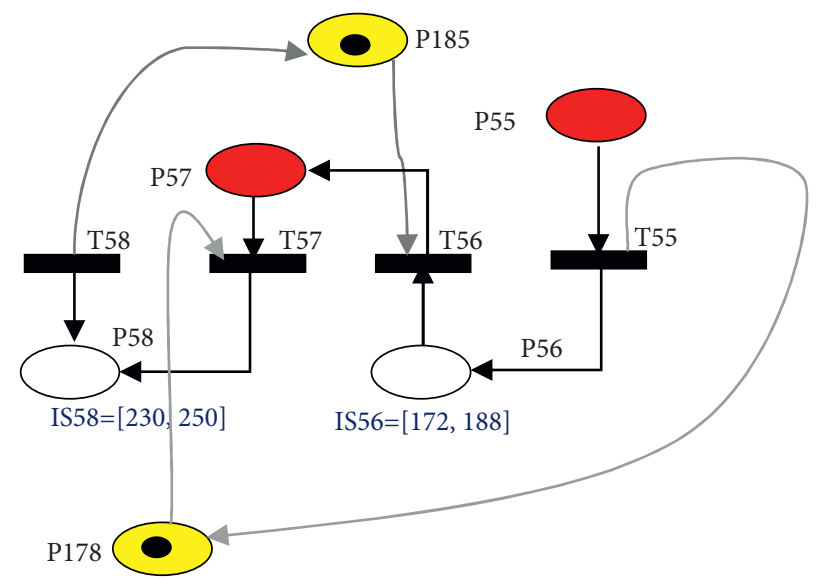

Figure 6: Catch-up places $P_{178}$ and $P_{185}$.

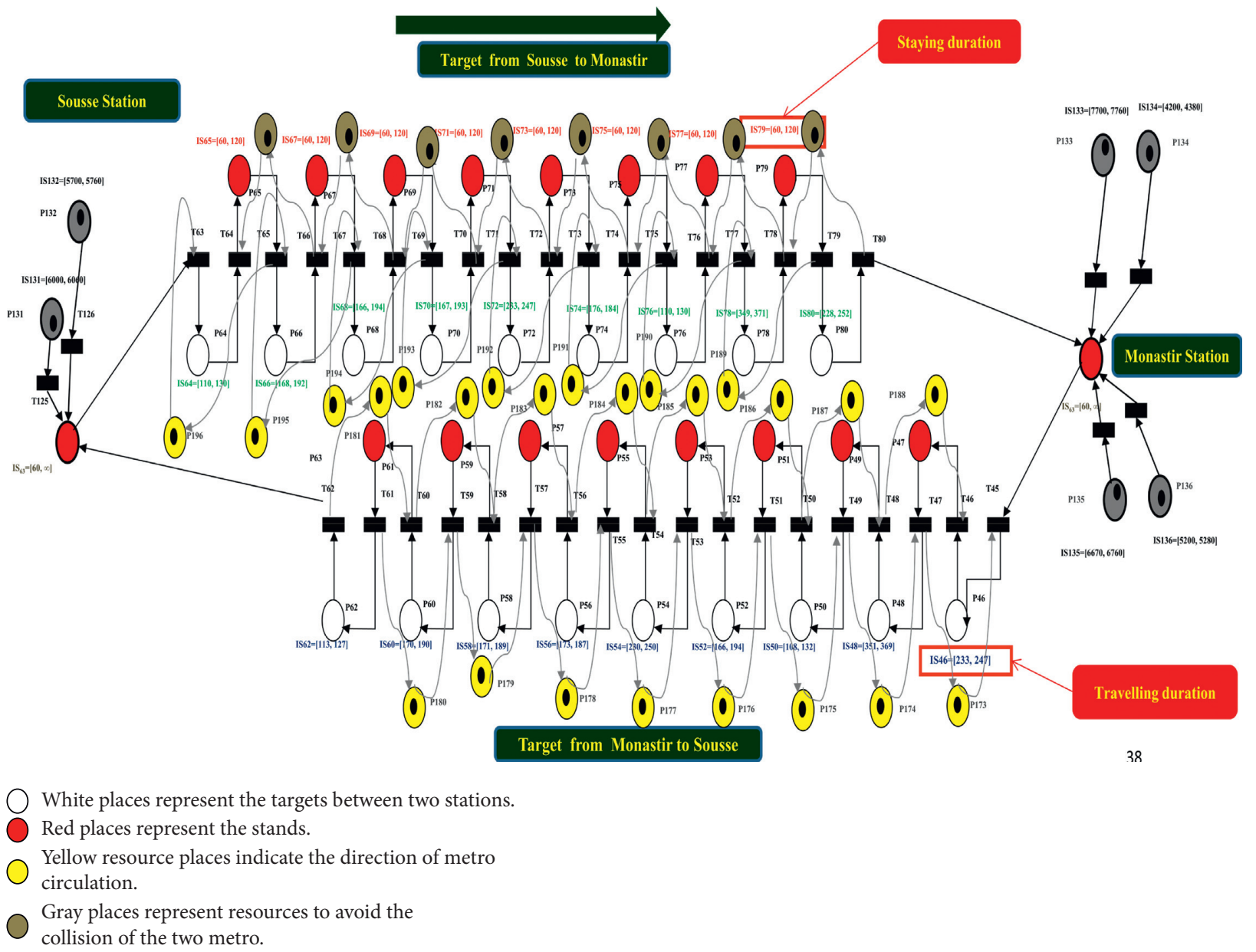

Figure 7: Stochastic P-time Petri net model for the Monastir-Sousse railway network.

point on the itinerary directly follows the exit point that precedes it. Coming back to the scheduling formalism, the itinerary can therefore be likened to a production range, and the scheduling is of the no-wait type. A no-wait constraint is inserted for any pair of consecutive operations in the itinerary and requires that these operations be processed without waiting time.

\section{Maintenance Scheduling in the Sahel Railway Network}

A maintenance scheduling problem is defined by a set of tasks (or operations), whose execution dates (start and end dates) are determined. This should generally be done by 
TABLE 2: Parking and travelling times.

\begin{tabular}{|c|c|c|c|}
\hline Place & $L_{i}$ & $H_{i}$ & $q e_{i}$ \\
\hline 45 & 58 & 62 & 60 \\
\hline 46 & 138 & 232 & 150 \\
\hline 47 & 38 & 42 & 40 \\
\hline 48 & 150 & 449 & 355 \\
\hline 49 & 28 & 32 & 30 \\
\hline 50 & 29 & 344 & 74 \\
\hline 51 & 28 & 32 & 30 \\
\hline 52 & 79 & 213 & 146 \\
\hline 53 & 38 & 42 & 40 \\
\hline 54 & 30 & 140 & 91 \\
\hline 55 & 28 & 32 & 30 \\
\hline 56 & 148 & 238 & 184 \\
\hline 57 & 28 & 32 & 30 \\
\hline 58 & 167 & 386 & 247 \\
\hline 59 & 28 & 32 & 30 \\
\hline 60 & 150 & 312 & 216 \\
\hline 61 & 28 & 32 & 30 \\
\hline 62 & 9 & 131 & 44 \\
\hline 63 & 48 & 52 & 50 \\
\hline 64 & 89 & 242 & 119 \\
\hline 65 & 28 & 32 & 30 \\
\hline 66 & 149 & 285 & 217 \\
\hline 67 & 28 & 32 & 30 \\
\hline 68 & 149 & 280 & 222 \\
\hline 69 & 28 & 32 & 30 \\
\hline 70 & 148 & 227 & 175 \\
\hline 71 & 28 & 32 & 30 \\
\hline 72 & 19 & 103 & 79 \\
\hline 73 & 38 & 42 & 40 \\
\hline 74 & 89 & 178 & 132 \\
\hline 75 & 28 & 32 & 30 \\
\hline 76 & 29 & 118 & 74 \\
\hline 77 & 28 & 32 & 30 \\
\hline 78 & 319 & 406 & 344 \\
\hline 79 & 38 & 42 & 40 \\
\hline 80 & 471 & 889 & 668 \\
\hline 131 & 28 & 32 & 30 \\
\hline 132 & 89 & 185 & 132 \\
\hline 133 & 28 & 32 & 30 \\
\hline 134 & 150 & 311 & 216 \\
\hline 135 & 28 & 32 & 30 \\
\hline 136 & 10 & 189 & 80 \\
\hline
\end{tabular}

optimizing a certain criterion and possibly assigning of a set of resources to the tasks.

A maintenance task is an entity defined by its start and end dates, its execution time, and its request for each resource. The problem can be preemptive if the accomplishment of a task can be done in several times. Otherwise, the problem is non-preemptive, and the task has to be performed only once.

To ensure rolling stock operation in the best conditions of safety and quality, the TNRC has specialized maintenance facilities responsible for the major repair and rehabilitation of rolling stock. These maintenance facilities provide high quality maintenance services and major repairs to the industrial customers.

In TNRC, each train should endure maintenance checks after a particular number of hours or distance (kilometers of travelling time). According to times and distances established by the regulations, maintenance procedures are divided into low and high levels. For high-level maintenance procedures (taking place every few months or years), metro systems are unavailable for routing purposes. These recovery procedures are sometimes performed at dedicated TNRC maintenance workshops.

For the lower level (every day or week), maintenance checks are performed at the corresponding TNRC depots, which have maintenance facilities among the network. In this paper, we will solely contemplate low-level maintenance within the tests conducted for this model.

Low-level maintenance could also be realized during a predefined maintenance time window at maintenance depots.

This time window limits the proportion of time and/or distance the metro will operate before the next compulsory 


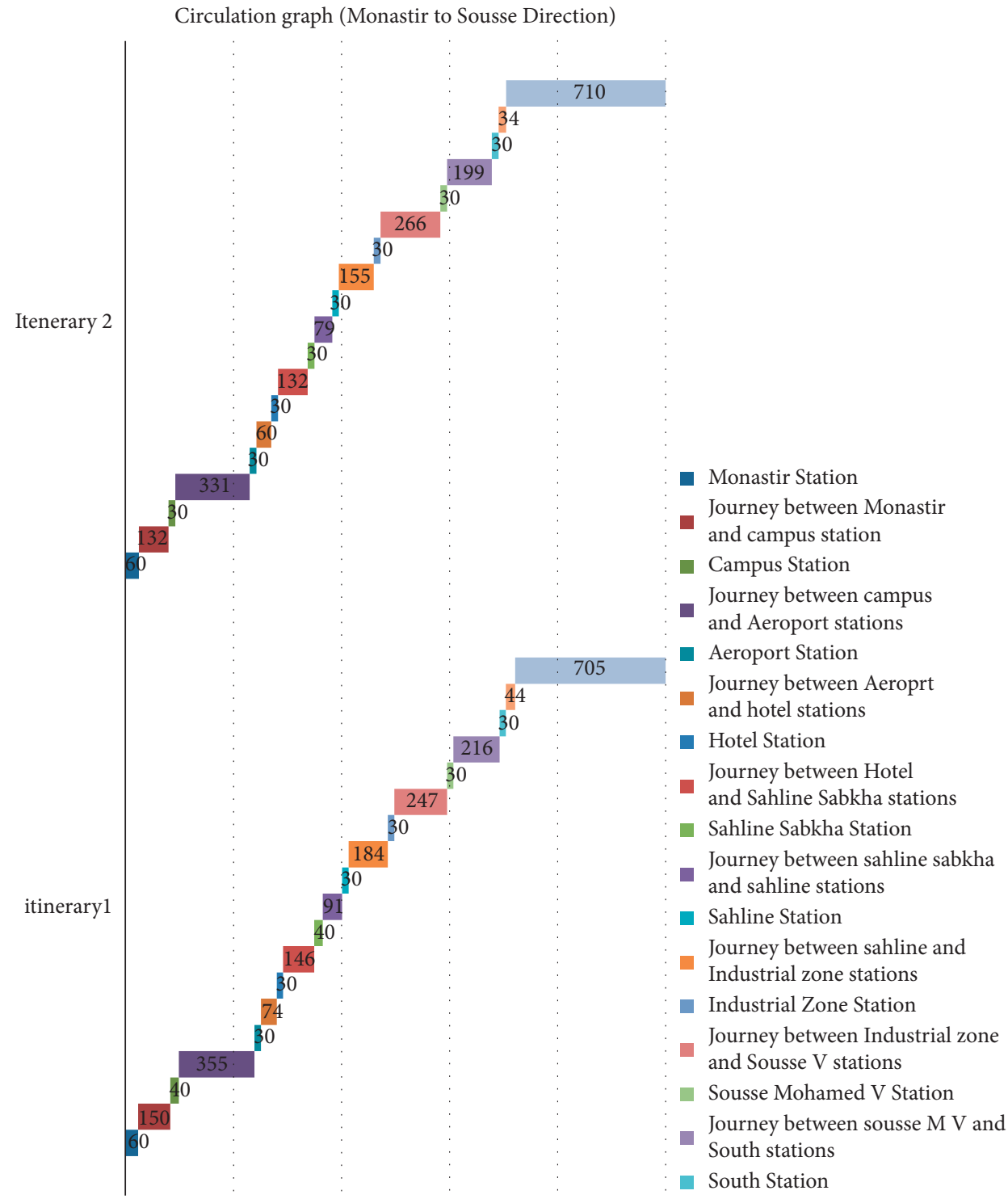

Figure 8: Circulation graph (Monastir to Sousse direction).

maintenance. The three low-level maintenance tasks consist of daily inspection (DI), weekly inspection (WI), and monthly inspection (MI). In the TNRC, a train must undergo a WI after operating for one or two weeks or for ten thousand kilometers before it can circulate again. A train must undergo DI after operating for two/three days. The inspections have a mean duration of one or two hours for the DI and of five to seven hours for the MI. These inspections are performed by an expert at the TNRC maintenance depots.

5.1. Static Integration of Maintenance Task. Let us take the example of itinerary 3, linking Sousse to Monastir station (Figure 10). Figure 10 represents the integration of additional jobs consisting of a sequence of maintenance operations (MOs). These operations represent the time allocated for the maintenance of the EMU (rolling stock) since it is available. The main objective is to try to take advantage of these periods of inactivity to start additional jobs such as predictive tasks (inspection, control, and so on). This method is referred to as static insertion which consists of the introduction of tasks when the metro is available without changing the initial scheduling solution. The introduction of the maintenance tasks must necessarily take into account the transport scheduling and time constraints.

In our study, we take under consideration that there are over twenty metro journeys between depots per day in the Sahel railway line. Thus, maintenance durations were reduced and only a short planning period is considered with a daily inspection, as shown in Table 3, where a sample solution for three trains extracted from the 10-trip model is given.

Given the maintenance railway scheduling, the mission of a railway operator is to determine the rolling stock routing which covers all the scheduled trips and minimizes the operating costs, considering the regulation on maximum travelling time and distance before maintenance checks. 


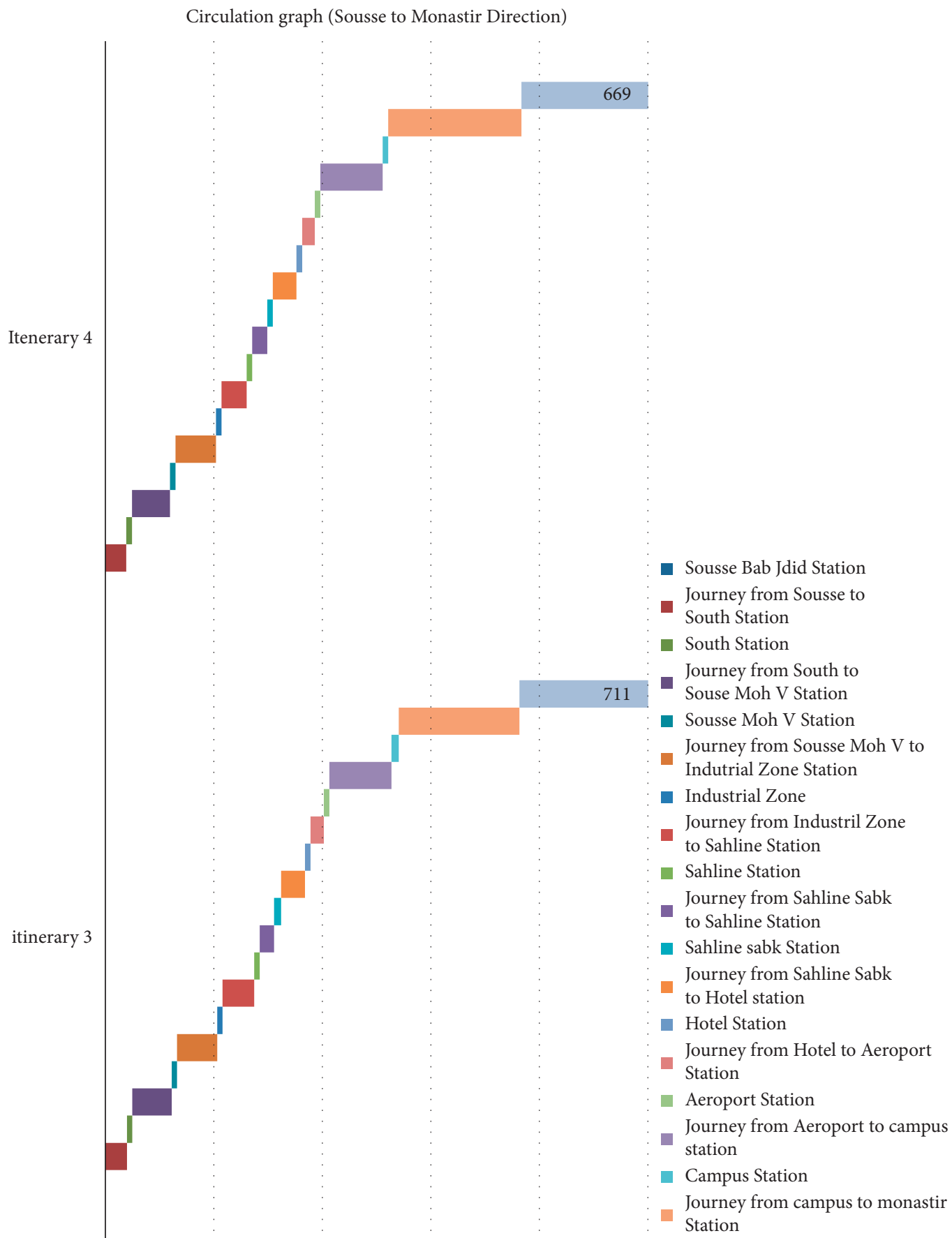

Figure 9: Circulation graph (Sousse to Monastir direction).

Considering that each train journey has been assigned, the maintenance scheduling seeks to determine the individual route taken by each train taking into account that the aforementioned maintenance constraints are checked.

5.2. Remarks. The proposed maintenance scheduling approach can be applied to any discrete event system integrating time constraints (urban, maritime, rail transport networks, manufacturing system with time constraints, and so on). The proposed approach would lead to more efficient inspection routines and directed anticipatory maintenance trips.

Certainly, the maintenance scheduling approach can be applied to other networks, but it does not cover three aspects:

The first is the progression of faults: it is not easy to define, extract, or "quantity" damage, but it may be worthwhile to consider the history of maintenance on a point and other relevant facts.

The second aspect is the deterioration process. It is not possible to wait until the component deteriorates to an 


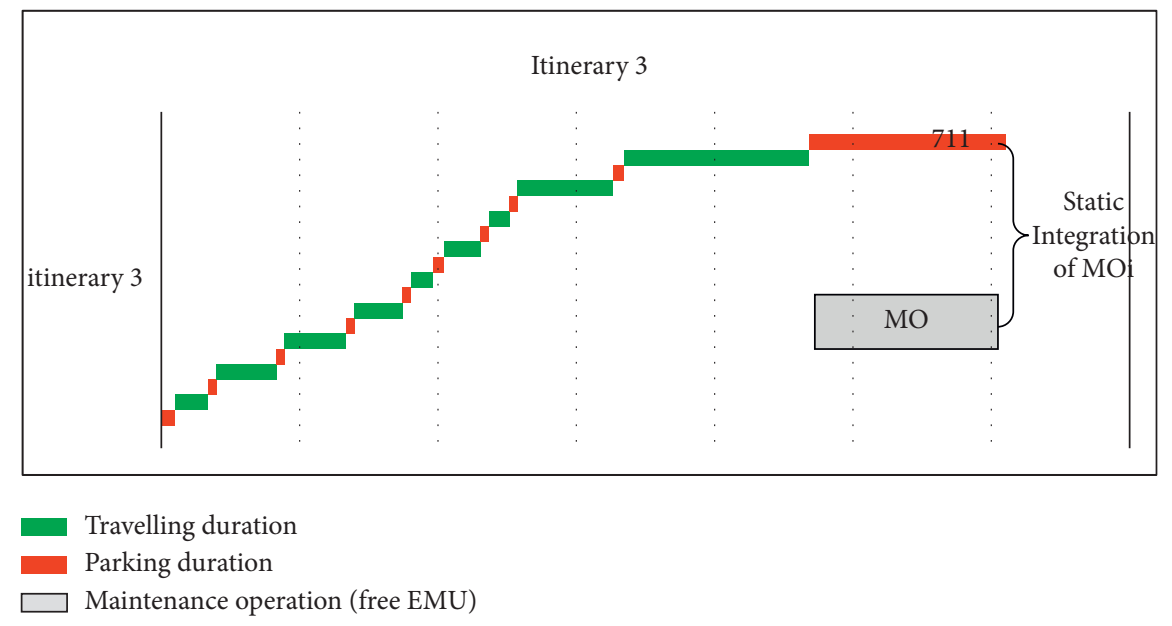

FIGURE 10: Maintenance scheduling of EMU.

TABLE 3: Example of performance tests (DI: daily inspection; TDI: total daily inspection; DDI: distance daily inspection).

\begin{tabular}{|c|c|c|c|c|}
\hline Train & Trip & DI $(\min )$ & TDI (min) & DDI $(\mathrm{km})$ \\
\hline 2 & 12 & 33 & 1052 & 32512 \\
\hline 3 & 28 & 64 & 6050 & 12174 \\
\hline 6 & 5 & 14 & 653 & 18143 \\
\hline \multicolumn{5}{|c|}{ Operation sequence } \\
\hline $\begin{array}{l}2 \\
3\end{array}$ & \multirow{2}{*}{\multicolumn{4}{|c|}{$\begin{array}{l}\text { Trip } 1 \text { at 21:00 from Store } 2->\text { Storage at } 21: 54 \text { in Depot } 3->\text { DI and Storage from } 22: 11 \text { to } 23: 01 \text { in Depot } \\
\text { Storage at } 7: 05 \text { in Depot } 1->\text { Trip } 3 \text { at } 8: 15 \text { from Store } 2->\text { DI and Storage from } 8: 55 \text { to } 9: 57 \text { in Depot } 2 \\
\text { Storage at } 10: 00 \text { in Depot } 2->\text { Trip } 5 \text { at } 11: 10 \text { from Store } 2->\text { DI + Storage from } 11: 14 \text { to } 13: 22 \text { in Depot } 1\end{array}$}} \\
\hline 6 & & & & \\
\hline
\end{tabular}

unrepairable status because it may be very unsafe when it is working in this condition. Hence, it would be better to repair or replace the components before its deterioration.

The third aspect is the maintenance resources (personnel, tools, drawings, spare parts, and so on). When a failure occurs and if drawings, maintenance teams, and tools are ready, the repair work can be started.

\section{Conclusions}

In this paper, we treated the scheduling problems of railway transport networks. This problem comes from the transport sector which requires immediate compliance with the operational safety rules. The aim is to insert additional recovery jobs in railway transport networks systems taking into account different scenarios and combinations of the scheduling service.

The proposed maintenance policy allows the avoidance of critical situations caused by disturbances. These disruptions can affect the railway infrastructure and traffic management and can lead to a degradation of the transport service. For adding maintenance tasks, we propose an inserting algorithm. In general cases, the established static integration allows the introduction of additional maintenance tasks in the availability of the machines without changing the initial scheduling solution. This strategy allows the traffic to continue in a degraded mode in the worst case. It is important to specify that the proposed approach is of the descriptive/predictive type usually specified through sets of equations and variables (as the cycle time of travelling circuits and the machine age) rather than of the decisional/ prescriptive type habitually specified through optimization models.

For the stage of adding maintenance tasks, we propose an inserting algorithm. The established static integration allows in the general case to introduce additional maintenance tasks in the availability of the machines without changing the initial scheduling solution. This strategy allows in the worst case to continue the traffic in a degraded mode. The presented scheduling approach allows solving problems of integration of the estimated or urgent jobs in planning, with the goal of minimizing the time and cost of traffic disturbance. The proposed insertion method shows how to integrate unforeseen maintenance actions and how to make real-time scheduling of these recovery acts with respect of transport scheduling constraint.

The proposed maintenance scheduling approach has been validated and verified by the real data provided by TNRC which manages the Sahel railway networks. Simulation results of the case study of a part of the Tunisian railway network show that method for integrating of recovery jobs in railway systems is able to modify the control models, activate urgent procedures, and decide about the selective maintenance task. The results obtained in the illustrative example 
are promising. They show that the proposed approach improves the prevention of temporal disruption and traffic management by performing early detection and can be used to evaluate the influence of different types of disturbances on the expected schedule.

6.1. Suggested Further Work. As further research, we are in the process of developing with the engineers of the railway company a platform (dashboard) allowing the monitoring and the selection in real time of the most appropriate maintenance strategy. The developed dashboard permits real-time data display and allows the supervisor to control the train locomotive through a graphical interface. The initial tests demonstrate that the developed approach has the potential to react in real time in order to avoid catastrophic scenarios and to improve rail safety.

Other research work carried out with TNRC engineers consists of incorporating the issues of maintenance and repair strategies into the maintenance scheduling approach to compute a modified maintenance cost. The development of operating and monitoring algorithms and their implementation on a PLC seem interesting for this rail transport application.

It would be attractive to apply the proposed scheduling approach to other issues, such as the scheduling of maintenance problems in urban transport networks, in order to attenuate the disruptions in the bus operating schedule. We intend, as future works, to propose a dynamic scheduling for railway transport systems, without foretelling moment of recovery task.

\section{Data Availability}

The data used to support the findings of this study are included within the article.

\section{Conflicts of Interest}

The author declares that there are no conflicts of interest regarding the publication of this paper.

\section{References}

[1] S. Bressi, M. Losa, and J. Santos, "Optimization of maintenance strategies for railway track-bed considering probabilistic degradation models and different reliability levels," Journal of Reliability engineering \& system safety, vol. 207, Article ID 107359, 2021.

[2] C. Cai, "Overview on safety management and maintenance of high-speed railway in China," Journal of Transportation Geotechnics, vol. 7, no. 2, Article ID 100397, 2020.

[3] L. Mira, A. R. Andrad, and M. C Gomes, "Maintenance scheduling within rolling stock planning in railway operations under uncertain maintenance durations," Journal of Rail Transport Planning \& Management, vol. 14, Article ID 100177, 2019.

[4] A. Consilvio, A. Di Febbraro, R. Meo, and N. Sacco, "Riskbased optimal scheduling of maintenance activities in a railway network," EURO Journal on Transportation and Logistics, vol. 8, no. 5, pp. 435-465, 2019.
[5] S. Deleplanque, "Maintenance on the railway network: disruptions and re-scheduling," Electronic Notes in Discrete Mathematics, vol. 69, pp. 109-116, 2018.

[6] P. C Gerum, A. Altay, and M. Baykal-Gürsoy, "Data-driven predictive maintenance scheduling policies for railways," Transportation Research Part C: Emerging Technologies, vol. 107, pp. 137-154, 2019.

[7] M. Yaghini, M. S. Mirghavami, and M. Shamaeezadeh, "A heuristic approach for scheduling of railway track maintenance machines," Quarterly Journal of Transportation Engineering, vol. 11, no. 3, pp. 557-576, 2020.

[8] I. Durazo-Cardenas, A. Starr, C. J. Turner et al., "An autonomous system for maintenance scheduling data-rich complex infrastructure: fusing the railways' condition, planning and cost," Transportation Research Part C: Emerging Technologies, vol. 89, pp. 234-253, 2018.

[9] S. Xie, C. Lei, and Y. Ouyang, "A customized hybrid approach to infrastructure maintenance scheduling in railroad networks under variable productivities," Computer-Aided Civil and Infrastructure Engineering, vol. 33, no. 10, pp. 815-832, 2018.

[10] J. Andrés, L. Cadarso, and Á. Marín, "Maintenance scheduling in rolling stock circulations in rapid transit networks," Transportation Research Procedia, vol. 10, pp. 524-533, 2015.

[11] Z. Su and B. Schutter, "Optimal scheduling of track maintenance activities for railway networks," IFAC-PapersOnLine, vol. 51, no. 9, pp. 386-391, 2015.

[12] C. Dao, R. Basten, and A. Hartmann, "Maintenance scheduling for railway tracks under limited possession time," Journal of Transportation Engineering, Part A: Systems, vol. 144, no. 8, Article ID 04018039, 2018. 